\title{
Separating fast and slow modes in coupled chaotic systems
}

\author{
M. Peña ${ }^{1}$ and E. Kalnay ${ }^{2}$ \\ ${ }^{1}$ SAIC at Environmental Modeling Center, NCEP, Camp Springs, Maryland, USA \\ ${ }^{2}$ Department of Meteorology, University of Maryland, College Park, Maryland, USA
}

Received: 29 September 2003 - Revised: 25 April 2004 - Accepted: 12 July 2004 - Published: 27 July 2004

\begin{abstract}
We test a simple technique based on breeding to separate fast and slow unstable modes in coupled systems with different time scales of evolution and variable amplitudes. The technique takes advantage of the earlier saturation of error growth rate of the fastest mode and of the lower value of the saturation amplitude of perturbation of either the fast or the slow modes. These properties of the coupled system allow a physically-based selection of the rescaling time interval and the amplitude of initial perturbations in the "breeding" of unstable modes (Toth and Kalnay, 1993, 1996, 1997; Aurell et al., 1997; Boffetta et al., 1998) to isolate the desired mode. We perform tests in coupled models composed of fast and slow versions of the Lorenz (1963) model with different strengths of coupling. As examples we present first a coupled system which we denote "weather with convection", with a slow, large amplitude model coupled with a fast, small amplitude model, second an "ENSO" system with a "tropical atmosphere" strongly coupled with a "tropical ocean", and finally a triply coupled system denoted "tropical-extratropical" in which a fast model (representing the "extratropical atmosphere") is loosely coupled to the "ENSO" system. We find that it is always possible to isolate the fast modes by taking the limit of small amplitudes and short rescaling intervals, in which case, as expected, the results are the same as the local Lyapunov growth obtained with the linear tangent model. In contrast, slow modes cannot be isolated with either Lyapunov or Singular vectors, since the linear tangent and adjoint models are dominated by the fast modes. Breeding is successful in isolating slow modes if rescaling intervals and amplitudes are chosen from physically appropriate scales.
\end{abstract}

\section{Introduction}

The Earth's weather and climate system contains chaotic subsystems spanning many different time scales, ranging

Correspondence to: M. Peña

(malaquias.pena.mendez@noaa.gov) from several minutes for individual convective clouds, to the seasonal-to-interannual phenomena associated with El Niño-Southern Oscillation (ENSO). Some of these subsystems are strongly coupled, as for example the tropical atmospheric/ocean system responsible for the ENSO chaotic behavior. Other chaotic subsystems are weakly coupled or not coupled at all. For example, mid-latitude weather is only weakly coupled with ENSO oscillations, and summer continental convection is weakly coupled (forced) by the local synoptic (large scale) waves, but independent of the largescale waves in other regions of the world.

Ensemble forecasting has the major goal of capturing the uncertainties of weather forecasts, but forecasts of phenomena with different time scales have different sources of uncertainties. In order to be able to represent forecast uncertainties, a forecast ensemble should include in the initial perturbations the type of dominant unstable perturbations responsible for forecast error growth and loss of skill. For example, a 6-h storm-scale forecast should include perturbations related to the fast instabilities of the mesoscale convective systems. In contrast, a three-day ensemble forecast should have within the initial perturbations the baroclinic instabilities of the evolving large-scale flow, and a seasonal forecast should include initial perturbations representing the even slower instabilities of the coupled ocean-atmosphere ENSO system.

These different types of instabilities cannot be all isolated with a linear system, such as the tangent linear and the adjoint models used to create Lyapunov Vectors (LVs) or Singular Vectors (SVs), since only the fastest instabilities dominate the growth rate in linear models. In fact, if it were possible to create a perfect model of the atmosphere that accounts for the motion of molecules, then Brownian motion would dominate the Lyapunov exponent computed from the linear tangent model.

Toth and Kalnay (1993) introduced the breeding method in order to create finite amplitude, finite time perturbations for ensemble forecasting. Breeding consists of adding an initial perturbation to a control run of a non-linear model, integrating forward in time, and periodically rescaling the 
amplitude of the perturbation by its magnitude at the end of the fixed rescaling time interval. The rescaled perturbation is added to the control run, and the process advances to the next time interval. Bred vectors (BVs) are the differences between the perturbed and the control runs and as such are a nonlinear generalization of the LVs (e.g. Toth and Kalnay, 1997; Kalnay et al., 2003). The local BV growth rate per unit time is defined as

$g(t)=\frac{1}{n \Delta t} \ln \left(|\delta \boldsymbol{x}| /\left|\delta \boldsymbol{x}_{\mathbf{0}}\right|\right)$,

where $n$ is the number of time integration steps $\Delta t$ of the rescaling interval, $\delta \boldsymbol{x}_{\mathbf{0}}$ is the bred vector at time $t_{0}$ and $\delta \boldsymbol{x}$ is the bred vector at the end of the breeding interval $\left(t=t_{0}+n \Delta t\right)$.

Toth and Kalnay (1993) showed that breeding, with rescaling time intervals of the order of a few hours to one or two days, could be used to estimate the shape of the baroclinic instabilities of the evolving background flow, if the amplitudes were chosen within the range of the analysis uncertainties $(1 \mathrm{~m}-15 \mathrm{~m}$ in the $500 \mathrm{hPa}$ geopotential height), yielding a typical hemispheric growth rate of about 1.5/day. However, if much smaller amplitudes were used (e.g. $1 \mathrm{~cm}$ ), the BVs grew an order of magnitude faster, and were clearly associated with convective instabilities dominating the tropics. They explained this result by the fact that convective instabilities grow faster, but saturate at a level much smaller than baroclinic instabilities. Toth and Kalnay $(1993,1997)$ pointed out that BVs, like the leading LVs, are independent of the norm used for rescaling, and depend only on the finite size of the initial perturbation. Toth and Kalnay (1993, 1996), and Kalnay and Toth (1996) conjectured that it was possible to use breeding to separate the LVs for a coupled system with fast and slow subsystem. Lorenz (1996) confirmed the validity of this conjecture for a low order model with a fast, small amplitude subsystem coupled to a slow, large-amplitude system.

Independently of this work, Aurell et al. $(1996,1997)$ proposed the use of a Finite Size Lyapunov Exponent (FSLE) in order to deal with the problem of multiple time scales and saturation of fast (and mostly irrelevant) instabilities. The FSLE is defined as:

$\lambda(\delta)=\frac{1}{\left\langle T_{r}(\delta)\right\rangle} \ln r$.

Here $T_{r}(\delta)$ is the time it takes for a perturbation with an initial size $\left|\delta x_{0}\right|=\delta$ to grow by a factor of $r$, chosen to be relatively small to avoid nonlinear saturation (e.g. if $r=2$, $T_{r}(\delta)$ is the doubling time), and $\langle\bullet\rangle$ represents an average over many realizations. The FSLE is obviously closely related to the average growth rate of BVs (1) per unit time:

$A B G R=\left\langle\frac{1}{n \Delta t} \ln \left(|\delta \boldsymbol{x}| /\left|\delta \boldsymbol{x}_{\mathbf{0}}\right|\right)\right\rangle$.

The difference between Eqs. (2) and (3) is that, in the computation of the FSLE, the growth factor $r$ is fixed and during the integration the variable time interval $T_{r}(\delta)$ needed to grow to this amplitude is measured. By contrast, in computing the average bred growth rate (ABGR), the interval between rescalings is fixed (for example by fixing the number of time steps $n$ ), and what is measured is the growth attained during the rescaling interval. Both the ABGR and the FSLE converge to the Lyapunov exponent in the limit of infinitesimal initial amplitudes and rescaling intervals. The ABGR may slightly underestimate the FSLE during periods in which the growth rate of the type of perturbations that are being considered is large, and nonlinear saturation may slow the growth. To avoid similar underestimations of the true growth rate, $\mathrm{Au}$ rell et al. (1997) recommended the use of small values of $r$, such as $\sqrt{2}$. The ABGR is easier to compute than the FSLE, and the local BGR (1) has been shown to be an excellent predictor of when regime changes will occur and how long they will last in the Lorenz (1963) model (Evans et al., 2004).

In current operational ocean-atmosphere coupled models for seasonal and interannual predictions (e.g. Bengtsson et al., 1993; Palmer and Anderson, 1994; Ji et al., 1996; Mason et al., 1999; Chang et al., 2000), perturbations for ensemble forecasting are introduced only in the atmosphere, and the ocean is not perturbed. Toth and Kalnay (1996) proposed that in the same way that convection could be considered as "noise" that saturates when dealing with baroclinic (weather) waves, breeding could be used in a coupled ocean-atmosphere model to identify the slow, coupled modes and separate them from "weather noise". Cai et al. (2003), pointed out that the coupled ocean-atmosphere instabilities of ENSO should be included in the perturbations if they are to create appropriate ensemble forecasts, and performed breeding in the Zebiak-Cane model representing ENSO instabilities (Zebiak and Cane, 1987). This provided the structure of the instabilities of the evolving ENSO background flow as a function of both the annual cycle and the ENSO phase. Cai et al. (2003) showed that the "spring barrier" in skill observed in both numerical and statistical forecasts started in the spring is due to the fact that the instabilities of the ENSO system grow fastest in the summer, and that if the initial errors do not project on the dominant bred vector, the spring barrier is essentially eliminated. These results were promising but they were obtained with an intermediate model in which the fast, chaotic atmosphere is replaced with a "slave" atmosphere in equilibrium with the ocean forcing, which implies that only slow modes are present in the solution.

Boffetta et al. (1998) used a weakly coupled fast/slow Lorenz model and the FSLE of Aurell et al. (1997) to show that the predictability time in a coupled system

$T_{p}=\int_{\delta}^{\Delta} \frac{d \ln \left(\delta^{\prime}\right)}{\lambda\left(\delta^{\prime}\right)} \approx \frac{1}{\lambda_{\max }} \ln \left(\frac{\Delta}{\delta}\right)$

is a function of the tolerance $\Delta$, and that for tolerances larger than the saturation level of the fast modes, the true predictability is larger than the Lyapunov estimation $T_{p}=\frac{1}{\lambda} \ln \left(\frac{\Delta}{\delta}\right)$ derived from the fast growth rate $\lambda$. In other words, if the fast modes have small amplitudes, their short predictability times are mostly irrelevant to the predictability implied by the slow modes. 
Although the results of Lorenz (1996), Cai et al. (2003) and Boffetta et al. (1998) are encouraging, it is not clear how to isolate slow ENSO unstable modes in a fully coupled fast/slow chaotic system, especially if the amplitude of the fast perturbations is not small compared with the slow perturbations, as is the case in the ENSO instabilities. Timmermann (2002) has suggested several possible approaches to address this problem, including the FSLE, and the replacement of the fast subsystem with a diagnostic "slave fast system", as done, for example in the Zebiak-Cane and other intermediate models. However, these approaches are not simple and it remains to be proven whether they work in a realistic case.

In this paper we introduce a straightforward approach to separate fast and slow instabilities in coupled systems based on the breeding technique in which the amplitude of the perturbation and the interval between rescalings are chosen based on physical scale considerations. The paper is organized as follows. In Sect. 2 we describe the basic models used in the experiments, in Sect. 3 we apply the mode separation to the basic "weather with convection" experiment, in which the fast modes are much smaller than the slow modes. We analyze the time series of the growth rate of the BVs, LVs and SVs. Similarly, in Sect. 4 we apply the mode separation to an ENSO-like coupled system, and to an additional experiment that mimics the coupling of a tropical ocean-atmosphere system with the extratropics. In Sect. 5, we discuss the results and the potential use of the technique in more realistic models.

\section{Saturation amplitude and time scales for two coupled Lorenz models}

To mimic the behavior of a coupled system with different time scales, we couple two versions of the Lorenz (1963) model, one designed to represent the fast subsystem and the other to represent the slow subsystem. The set of equations are

$$
\begin{aligned}
\dot{x} & =\sigma(y-x)-c\left(S X+k_{1}\right) \\
\dot{y} & =r x-y-x z+c\left(S Y+k_{1}\right) \\
\dot{z} & =x y-b z+c_{z} Z \\
\dot{X} & =\tau \sigma(Y-X)-c\left(x+k_{1}\right) \\
\dot{Y} & =\tau r X-\tau Y-\tau S X Z+c\left(y+k_{1}\right) \\
\dot{Z} & =\tau S X Y-\tau b Z-c_{z} z,
\end{aligned}
$$

where capital letters represent the slow system, $\sigma=10$, $b=8 / 3$, and $r=28$ are the standard values of the Lorenz model parameters, $c$ is the coupling strength of the $x$ and $y$ components, $c_{z}$ is the coupling strength of the $z$ component, and $k_{1}$ is an "uncentering" parameter taken to be 10 except in the "ENSO" experiment where it is -11 . When $c=c_{z}=0$ the original Lorenz model is recovered. The parameters $S$ and $\tau$ represent the spatial and temporal scale factors, respectively. In this coupling $S=0.1$ implies that the slow system amplitude scale is chosen to be 10 times larger than the fast system; $S=1$ that they have the same amplitude scale (although the actual scale depends on the details of the coupling), and $\tau=0.1 \mathrm{implies}$ that the slow system is 10 times slower than the fast. Some cases considered in this section are given in Table 1.

We assign a name to each of the cases suggesting a qualitative association with coupled modes that exist in the climate system, with convection having a small amplitude and weak coupling with the underlying weather (baroclinic) waves, a weak extratropical coupling between the atmosphere and the ocean, and a strong atmosphere-ocean coupling simulating the tropical El Niño-Southern Oscillation (ENSO). In Sect. 4 the "ENSO" system is further coupled with an "extratropical atmosphere". Equations (5) are integrated using a fourth order Runge-Kutta time scheme with a time step of $\Delta t=0.01$. The initial transient period (first $2^{12}$ steps) is discarded. In the computation of growth rates of BVs, SVs, and LVs, we used the same initial conditions.

As described in Sect. 1, the two parameters that can be "tuned" in breeding are the initial amplitude of the perturbation $\delta=\left|\delta \boldsymbol{x}_{\mathbf{0}}\right|$ and the length of the rescaling period $n \Delta t$. For the computation of the leading LVs, we used $\boldsymbol{L}$, the tangent linear model of (Eq. 5) integrated over the same interval used for rescaling in the breeding experiments. We computed the leading SVs as the first eigenvector of $\boldsymbol{L}^{T} \boldsymbol{L}$ using the Eulerian norm, and its growth rate is the square root of the corresponding eigenvalue (e.g. Kalnay, 2003).

In order to establish the characteristic scales of error saturation, we performed 60-member ensembles of nonlinear forecasts started with small initial random perturbations (5\% of the typical variability of each system) for the cases in Table 1. Figure 1 shows the average distance among ensemble members, which can be interpreted as a measure of the typical size of saturation errors of the system, bounded by the size of the attractor in the phase space. In the "weather with convection" case, the solutions of both fast and slow systems in phase space (Fig. 2a) have similar behavior but the slow variability is an order of magnitude larger in both time and space. These differences in time and spatial scales are reflected in the ensemble mean distance (Fig. 1a), which indicates that the fast modes reach much faster the size and time of saturation. This is not surprising because the coupling is relatively weak and because, according to (Eq. 5), the same dynamic applies to both subsystems. This situation does resemble the large amplitude, slow perturbations associated with atmospheric baroclinic instability coupled with the small and fast perturbations associated with atmospheric convection, and in fact, Fig. 1a is similar to the schematic Fig. 6 that Toth and Kalnay (1993) used to explain why the use of very small amplitudes led to the recovery of modes related to convection, rather than to the more energetic baroclinic modes obtained with larger amplitudes. It shows that the distance between two randomly chosen runs reaches a maximum value (saturates) in the first few hundreds time steps for the fast component, whereas the slow component reaches saturation after about 8000 time steps. Figure 1a suggests that both the earlier saturation and the smaller size of the amplitude reached after saturation could be used to define 
Table 1. Representative coupling cases and parameters in model given in Eq. (1). The timescale is $\tau=0.1$ for all the experiments.

\begin{tabular}{llll}
\hline Type of coupling & Coupling strength & Amplitude scale & Offset \\
\hline "Weather/convection" & $c=0.15, c_{z}=0$ & $\mathrm{~S}=0.1$ & $k_{1}=10$ \\
"Extratrop. Ocean/Atm. 1" & $c=0.15, c_{z}=0$ & $\mathrm{~S}=1$ & $k_{1}=10$ \\
"Extratrop. Ocean/Atm. 2" & $c=0.08, c_{z}=0$ & $\mathrm{~S}=1$ & $k_{1}=10$ \\
"ENSO" & $c=1, c_{z}=1$ & $\mathrm{~S}=1$ & $k_{1}=-11$ \\
"ENSO/extratropical" & $c=c_{z}=1, c_{e}=0.08$ & $\mathrm{~S}=1$ & $k_{1}=10, k_{2}=-11$ \\
\hline
\end{tabular}
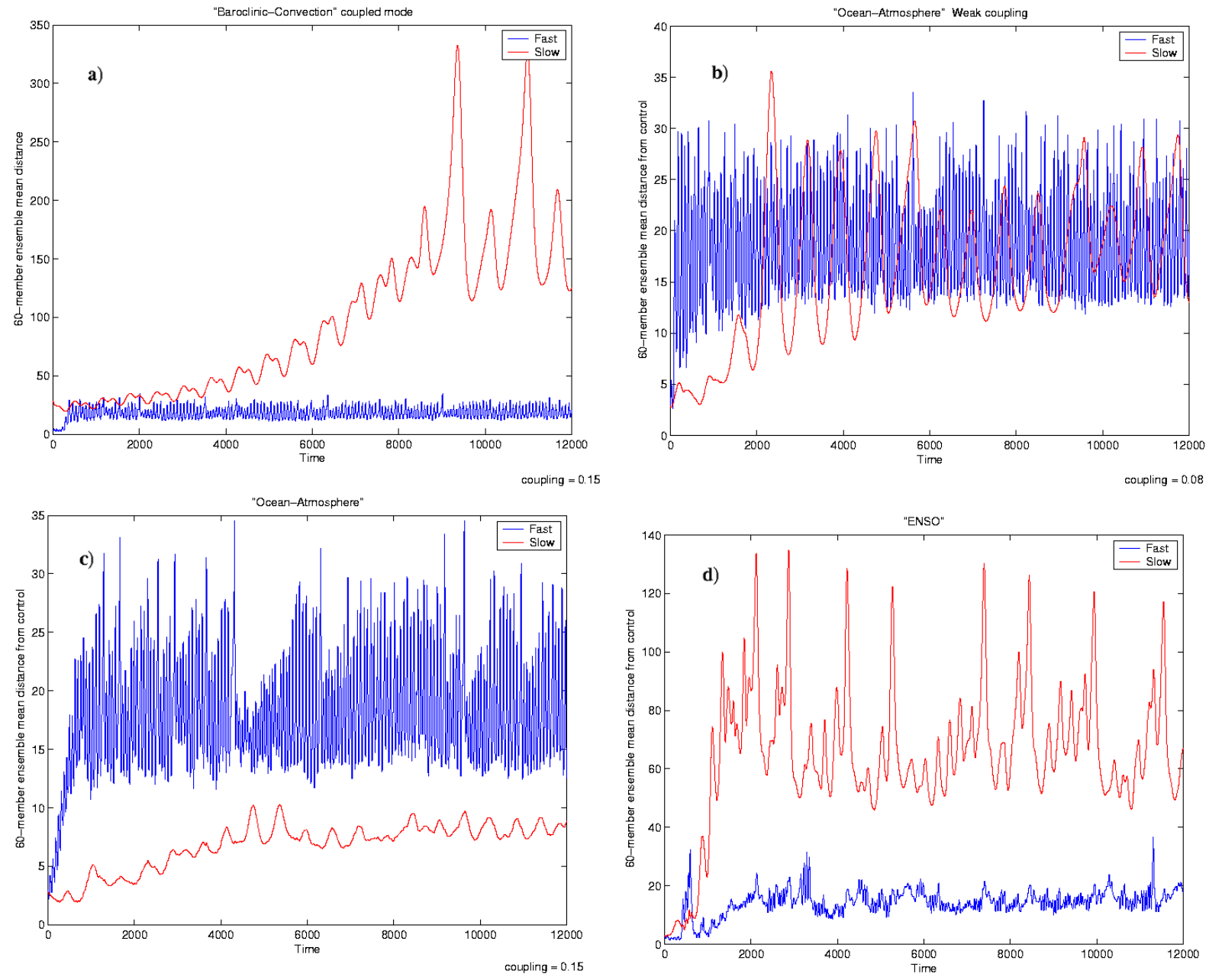

Fig. 1. Evolution of the rms distance of ensemble members for runs corresponding to the first four cases given in Table 1, and random small initial perturbations.

a suitable approach to filter out the fast modes and extract the slow growing modes in the coupled system.

For the "extratropical ocean-atmosphere" cases, Figs. 1b and $1 \mathrm{c}$, it is clear that the coupling strength modifies their relative amplitude even though we used a unit scaling factor $S=1$. When the coupling is very weak as in Fig. 1c, their amplitudes are nearly the same, but with a somewhat stronger coupling, as in Fig. 1b, the amplitude of the slow "ocean" is considerably reduced, and it becomes a "slave" of the fast "atmosphere". These cases qualitatively represent the situation of an extratropical ocean coupled with the atmosphere, in which atmospheric weather variability has shorter time scales and larger amplitudes than the atmospheric variability associated with ocean coupling. These figures suggest that it is not possible to filter out the fast modes by simply choosing large amplitudes of initial perturbations as in the "baroclinic-convective" case. Yet, the fast mode still saturates earlier than the slow mode and, with relatively weak 

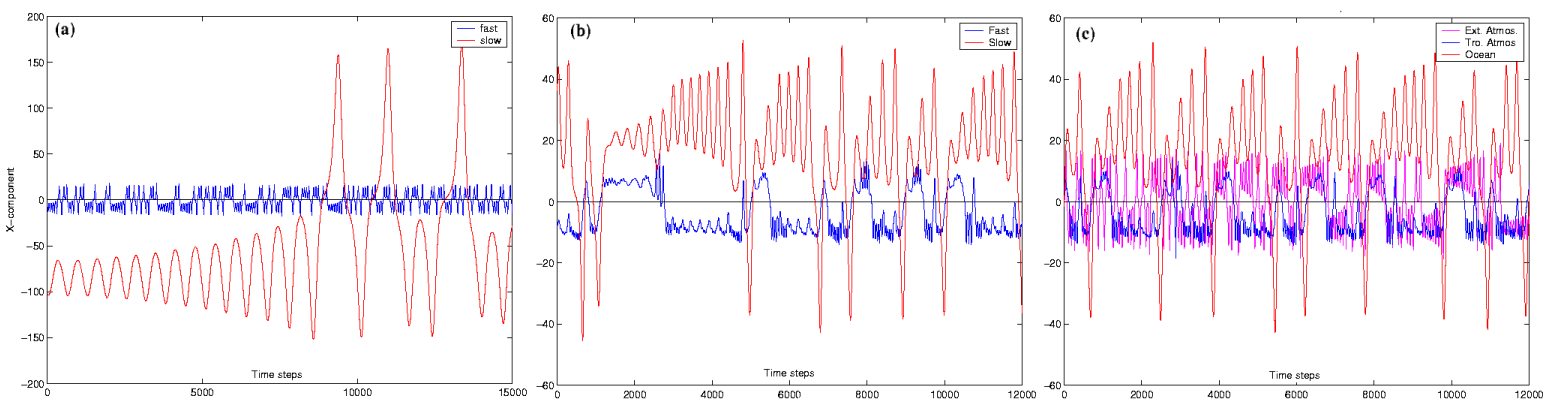

Fig. 2. Evolution of the $x$-component of each of the systems of the coupled model: slow and fast systems for (a) the "weather waves with convection", (b) the "ENSO", and (c) the "Extratropical Atmosphere", "Tropical Atmosphere" and the "Ocean" sub-systems in the "Tropics-extratropics" coupled model.
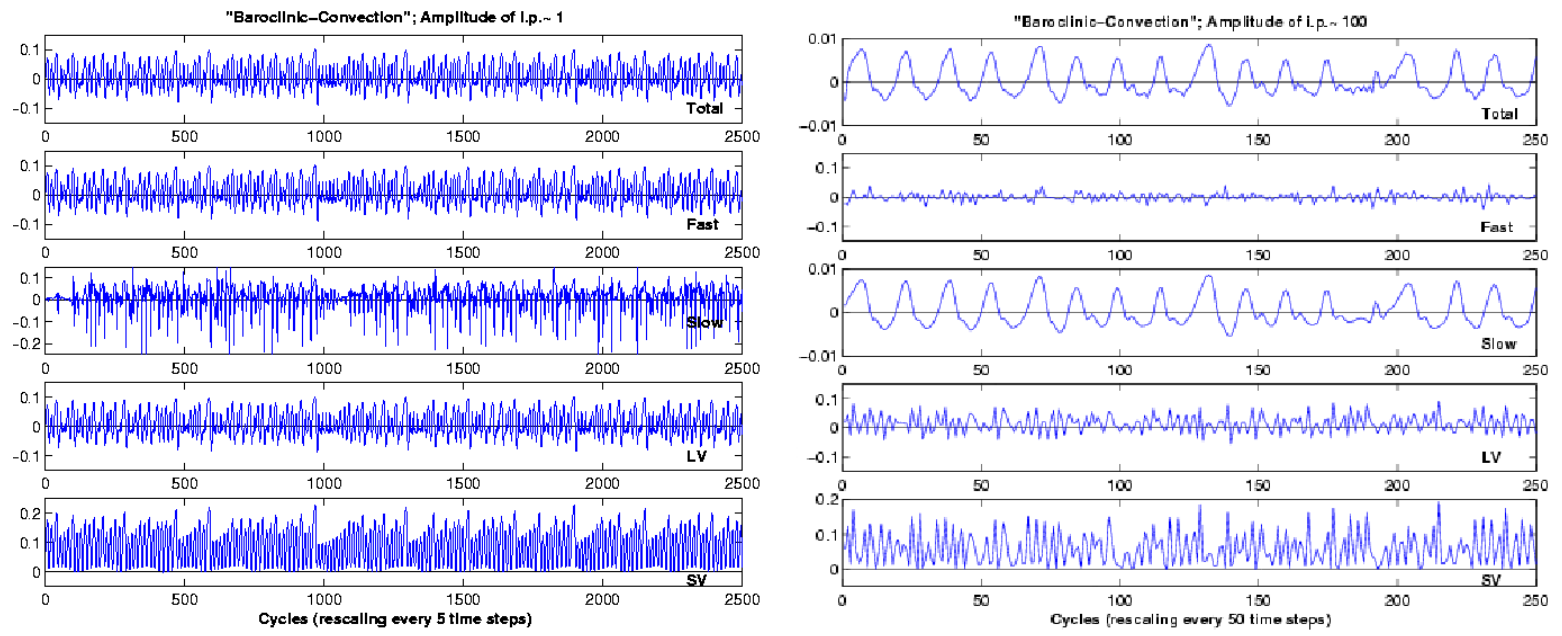

Fig. 3. Left panel: Growth rate of (a) the total BV, (b) fast component, and (c) slow component, (d) LV and (e) SV, (left) using initial perturbations of 1 for the case of the "weather waves with convection" shown in Fig. 1a, and rescaling every 5 time steps. Right panel: same as left panel but using initial perturbations of amplitude 100 and rescaling intervals of 50 time steps. The results are insensitive to small variations in the amplitude or rescaling interval. Notice that the range is different for the growth rate of the total and the slow

coupling, the two modes evolve with time scales much different from each other. Therefore, choosing a rescaling interval sufficiently long to allow saturation of the fast mode could reduce its impact on the error growth rate of the coupled system. In the "ENSO" case (Fig. 1d) we used much stronger coupling $(c=1)$ and coupled the z-component as well, using $c_{z}=1$. The result is a time-series with a slow mode that shift regimes every 3 to 12 cycles and a fast mode with more irregular behavior (Fig. 2b). Since the coupling is very strong, the saturation time is about the same for the fast and slow solutions. Note that even though we used again a scaling factor $S=1$, in this case the fast solution saturation amplitude is smaller, and the variability lower than in an uncoupled fast system, a result reminiscent to the tropical coupled ENSO response.

In the next two sections we present detailed comparisons of fast and slow growth for the "weather with convection" case, for the "ENSO" case, and for a triply coupled system, in which the tropical atmosphere of ENSO is weakly coupled with an "extratropical" atmosphere. The other two cases presented in Fig. 1 are less interesting for the following reasons. In the case of Fig. 1b, the slow ocean is driven by the atmosphere, and provides only a weak feedback, and therefore there are no important slow modes dominating the coupled dynamics. In the case of Fig. 1c, for this configuration of parameters, the two systems are essentially independent, and breeding for the atmosphere and the ocean can be done independently from each other, rescaling with the corresponding shorter or longer intervals.

\section{Separation of the fast and slow modes in the "weather with convection" coupled case}

Based on the results of Figs. 1a and 2a, we chose physically based amplitudes and rescaling intervals in order to separate the fast and slow coupled modes for the case of small fast "convective" modes coupled with large, slow "weather" waves. The results are presented in terms of the local bred 

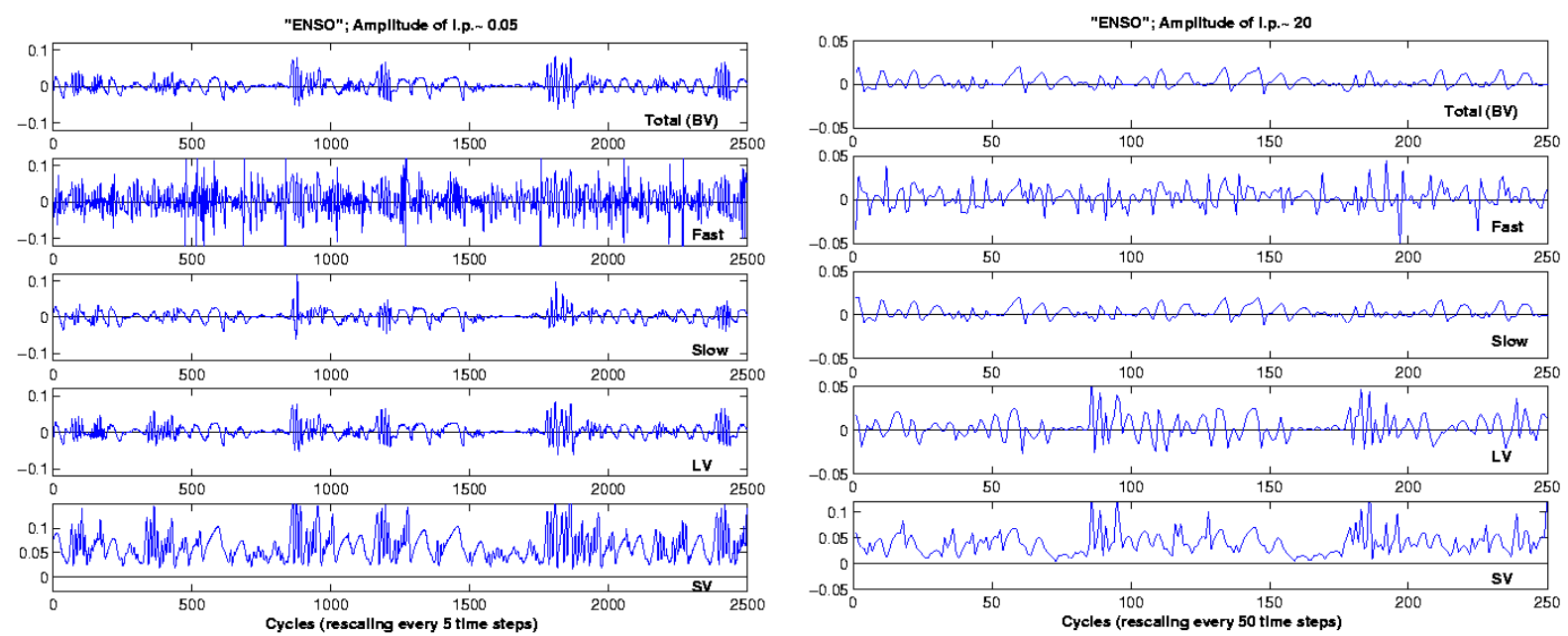

Fig. 4. Same as Fig. 3 but for the coupled "ENSO" system shown in Fig. 1d. The left panel shown the results obtained using a perturbation size 0.05 , and rescaling every 5 steps, and the right panel, results obtained using a perturbation size 20 and rescaling every 50 time steps. The results are insensitive to small variations in the amplitude or rescaling interval.

growth rate (Eq. 1) in Fig. 3, with the left panels corresponding to a small amplitude $\delta=1$ and a short rescaling interval $5 \Delta t$, and the right panels to a large amplitude $\delta=100$ and a long rescaling period $50 \Delta t$. The top 3 panels correspond to the BVs growth rate computed with the nonlinear model, and the bottom two panels to the LVs and SVs growth rate over the same period, computed with linear tangent model and its adjoint (transpose). In the top three panels we plot the total coupled growth rate (labeled as "total"), the growth rate measured with just the fast variables ("fast"), and the growth rate measured with the slow variables ("slow"). The abscissa is labeled in number of rescaling intervals, but the left and right panels always correspond to the same elapsed physical time.

Considering first the left panel, with small amplitudes and short rescaling interval, we see that the growth of the fast "convection" mode is clearly identified, and dominates the total growth. As expected, the growth rate obtained with the linear tangent model (Lyapunov exponent) is also dominated by the fast modes and is essentially identical to that obtained with the BVs. Also as expected, the growth rate of the SVs is considerably larger than that of the LVs. The SV growth rate oscillates with the same frequency as the LV growth rate, but its minimum value is modulated by a frequency associated with the slow modes growth rate (cf. right panel).

The right panels are constructed with parameters chosen to identify the slow "weather waves", using a large amplitude and long rescaling intervals. The top three panels indicate that the breeding approach succeeds in isolating the slow modes. The slow growth is apparent when measuring the growth with the slow variables, and it clearly dominates the total growth rate, although during those intervals in which the slow modes decay (growth less than zero), there is a perceptible influence of the fast modes on the total rate, modulating the rate of decay. In this case, the bottom two panels show that the linear model-based LV and SV growth rates fail to capture the growth rates of the high amplitude, low frequency "weather waves", and instead they are still dominated by the high frequency growth of the "convective modes". It is interesting to note that for the longer rescaling, the SVs growth rates are larger but become strongly correlated with the LVs growth rate. This is because the SVs are "optimized" for the rescaling period, and as the optimization period increases, the evolved (final) SVs become parallel to the LVs and grow at a similar rate (e.g. Legras and Vautard, 1997).

These results support the conjecture of Toth and Kalnay (1993, 1996) and Kalnay and Toth (1996), that the selection of rescaling amplitude and frequency could be used to separate fast and slow modes, when the latter have larger amplitudes. It also confirms the results obtained by Lorenz (1996), and is also in agreement with the results of Aurell et al. (1997) and Boffetta et al. (1998). In the next section we tackle more difficult cases in which the scaling factor $\mathrm{S}$ is chosen to be unity.

\section{Cases of "ENSO" and "ENSO coupled with an extra- tropical atmosphere"}

a) "ENSO" case

As we saw in Sect. 2, the strongly coupled ENSO-like case results in an almost "slave" atmosphere with a small amplitude and regime changes clearly modulated by the slow "ocean" component (Fig. 2b). Figure 4 shows the same panels as Fig. 3, but for the ENSO case. On the left, when we use small amplitudes $(\delta=0.05)$ and short rescaling intervals $(5 \Delta t)$, we find that most of the time the growth measured by the slow variables still dominates the total growth. As a result, the total growth rate takes place mostly on long time scales except for brief intervals of fast growth. In this case, the LV growth rate is quite similar to the growth rate measured with the slow variables, and is virtually identical to the 

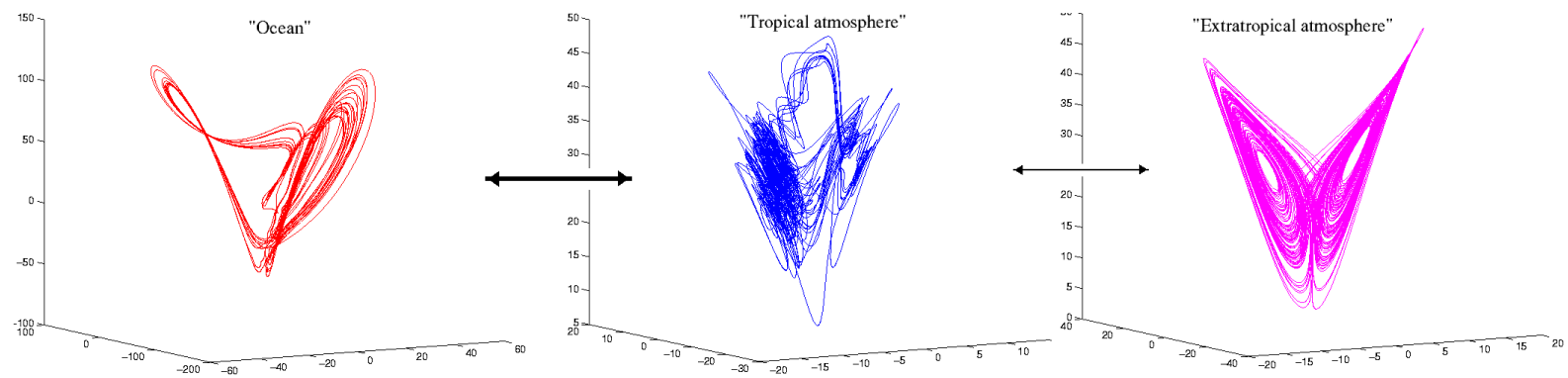

Fig. 5. Attractors of the 3-component "tropical-extratropical" coupled system corresponding to Fig. 2c. The "tropical ocean" (left) is strongly coupled to the "tropical atmosphere" (center), which in turn is weakly coupled to the "extratropical atmosphere" (right). The thickness of the arrows indicates the strength of the coupling.
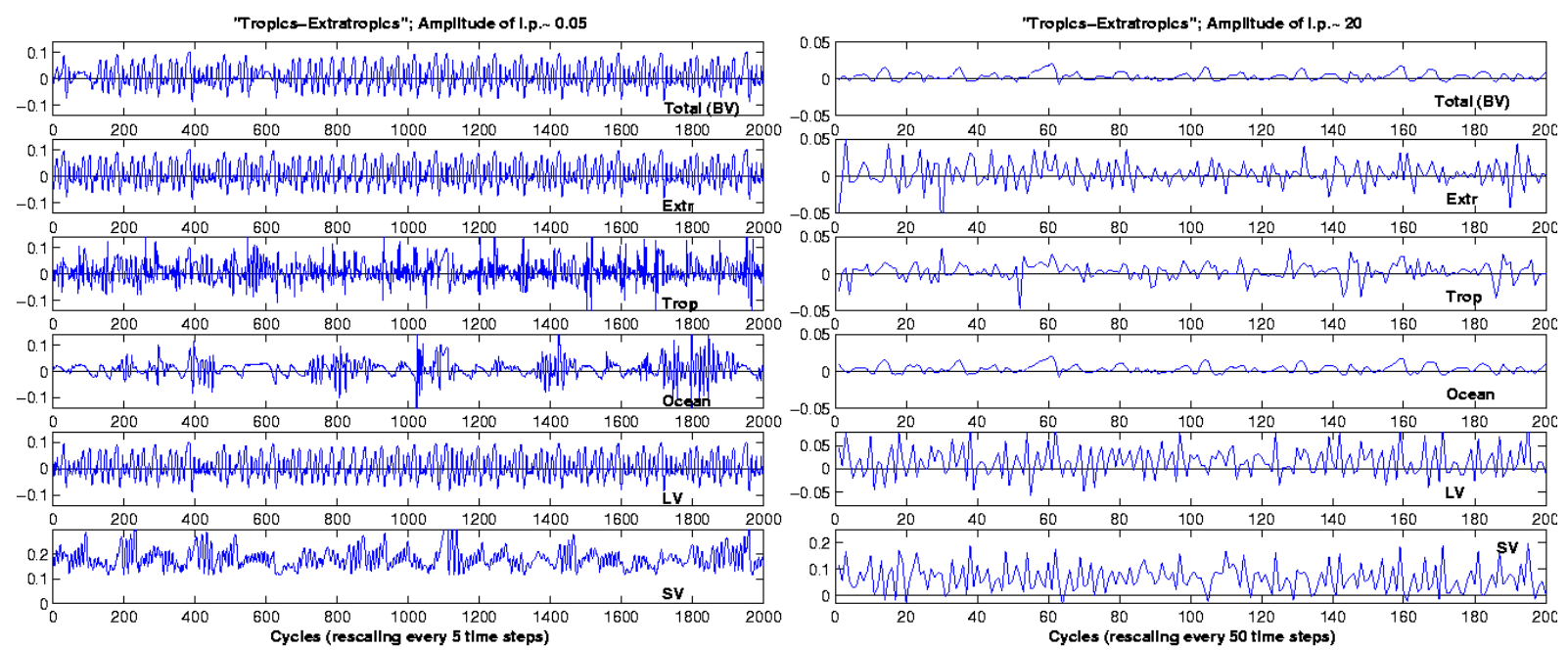

Fig. 6. Same as Fig. 3 and Fig. 4 but for the three component "tropics-extratropics" of Figs. 2c and 5. The rescaling parameters are the same as in Fig. 4.

total BV growth rate, as it should, since the BVs coincide with LVs in the limit of infinitesimal amplitudes and rescaling intervals. For this case even the SV growth rate is dominated most of the time by the slowly evolving scales. On the right panels, we see the results obtained using larger amplitudes $(\delta=20)$ and longer rescaling periods $(50 \Delta t)$. The results for the bred vectors show that the fast growth spurts have been filtered out from the slow and total growth rates, but otherwise there is still a similarity, which is to be expected given the secondary, almost diagnostic role of the atmosphere in this coupled system. On the other hand, the growth rates for the LVs and SVs are still similar to those obtained at low amplitudes and high frequency rescaling (left panels). The spurts of fast growth are still present at the same time, and are only smoothed to the extent that their frequency is higher than that of the rescaling, indicating that the linearized models still responds to the same instability characteristics. Additional experiments (not shown) indicate that for initial perturbations above $\delta=20$ the slow mode has the same temporal structure independently of the rescaling time period, confirming that the slow and total bred vectors have a growth that depends on the stability of the background flow and not on the details of the parameters used for breeding.

b) "Extratropical atmosphere coupled with ENSO"

This is the most complex example we will use as illustration of the impact of using different amplitudes and scaling intervals. In this case we add an "extratropical atmosphere" which is weakly coupled with the "tropical atmosphere" component of "ENSO". The equations for this system are as follows:

$$
\begin{aligned}
& \dot{x_{e}}=\sigma\left(y_{e}-x_{e}\right)-c_{e}\left(S x_{t}+k_{1}\right) \\
& \dot{y_{e}}=r x_{e}-y_{e}-x_{e} z_{e}+c_{e}\left(S y_{t}+k_{1}\right) \\
& \dot{z}_{e}=x_{e} y_{e}-b z_{e} \\
& \dot{x_{t}}=\sigma\left(y_{t}-x_{t}\right)-c\left(S X+k_{2}\right)-c_{e}\left(S x_{e}+k_{1}\right) \\
& \dot{y_{t}}=r x_{t}-y_{t}-x_{t} z_{t}+c\left(S Y+k_{2}\right)+c_{e}\left(S y_{e}+k_{1}\right) \\
& \dot{z}_{t}=x_{t} y_{t}-b z_{t}+c_{z} Z \\
& \dot{X}=\tau \sigma(Y-X)-c\left(x_{t}+k_{2}\right) \\
& \dot{Y}=\tau r X-\tau Y-\tau S X Z+c\left(y_{t}+k_{2}\right) \\
& \dot{Z}=\tau S X Y-\tau b Z-c_{z} z_{t}
\end{aligned}
$$

In these equations lower cases represent variables corresponding to the fast atmospheres, with the extratropical 
atmosphere (denoted with a subscript $e$ ) weakly coupled to the tropical atmosphere (with a subscript $t$ ), which in turn is strongly coupled to the ocean (upper case variables) as in the ENSO case. The tropical atmosphere and ocean variables are fully coupled with a coupling coefficient $c=c_{z}=1$, as in the ENSO case, and the extratropical atmosphere is coupled to the tropical atmosphere with a weak coupling coefficient $c_{e}=0.08$. Figure 5 shows the shape of the coupled attractor for each of the components. On the left is the slow ocean, vacillating between a "normal" state which lasts typically 3 to 12 "years", and an "El Niño" state, which lasts only one "year" (see also Fig. 2c, presenting the x component of the system versus time). In the center is the "tropical ENSO atmosphere", faster but strongly coupled to the ocean, as in the ENSO case. On the right is the "extratropical atmosphere" only weakly coupled to the tropical atmosphere, and therefore looking closer to the classic Lorenz model.

Figure 6 is similar to Fig. 4, but for the tropicalextratropical coupled system. On the left we see that for small amplitudes $(\delta=0.05)$ and short rescaling intervals $(5 \Delta t)$, the growth rate of the extratropical atmosphere dominates the total growth rate, as could be expected because the parameters are appropriate for this system. Once again, for small amplitudes and frequent rescalings, the $\mathrm{LV}$ growth rate is almost identical to the total growth rate, dominated by the extratropical atmosphere, and the growth rate of the SVs is very large and less variable. For an even shorter rescaling interval, the results are similar except for the growth rate of the SVs, which becomes much larger and almost constant, suggesting that it cannot discriminate between periods of real growth and decay in the extratropical atmosphere.

On the right, we see that for longer rescaling periods $(50 \Delta t)$ and larger amplitudes $(\delta=20)$, the fast extratropical atmosphere oscillations are essentially completely filtered out, and the ocean growth rate dominates the tropical atmosphere, as well as the total growth rate. Unfortunately, the linear approaches of the LV and SV growth, although similar to each other, are strongly influenced by the extratropical solutions, and do not provide perturbations appropriate for the longer time scales.

\section{Summary and discussion}

We have shown that a simple generalization of breeding using amplitudes and rescaling intervals that are physically chosen can be used to separate slow and fast solutions in coupled systems. It should be noted that the results obtained for the BVs and LVs are not sensitive to small variations of the two parameters as long as they are within the range suggested by the amplitude and time scales of the coupled solution (Fig. 1). The growth rates for the SVs, by contrast, are considerably more sensitive. The growth rates of the BVs reflect the stability of the basic, evolving flow at the corresponding time scales. In addition to their growth rate, this approach yields the bred vectors perturbations appropriate for different types of ensemble forecasts.
The results suggest that for realistic atmospheric models, frequent rescaling (of the order of 10 minutes) and small amplitudes in the temperatures and other variables could be used to obtain bred perturbations in "storm-scale" models. For large-scale weather forecasting, amplitudes of the order of $1-10 \mathrm{~m}$ in geopotential heights and intervals of $6-48 \mathrm{~h}$ have been already shown to be successful in creating baroclinic initial perturbations. For seasonal and interannual predictions, whose skill depends strongly on capturing the evolution of ENSO variability, Cai et al. (2003) have suggested that rescaling intervals of the order of two weeks to a month may isolate coupled model instabilities, and Cai et al. (2003) and Yang et al. (2003) presented results that suggest that this is indeed the case.

Finally, we point out that since there is a relationship between breeding and ensemble Kalman Filtering (e.g. Corazza et al., 2002), our results suggest that for data assimilation in a coupled ocean-atmosphere system, the interval between analyses should be chosen in a similar fashion, allowing enough time for the fast but irrelevant atmospheric oscillations to saturate, and not to overwhelm the slower but important growth rates of the coupled ENSO instabilities.

Acknowledgements. This research was supported by the NOAAOffice of Global Programs. The authors thank J. Hansen (MIT) for providing the original version of the Lorenz coupled model.

Edited by: A. Osborne

Reviewed by: one referee

\section{References}

Aurell E., Boffetta, G., Crisanti, A., Paladin, G., and Vulpiani A.: Predictability in the large: an extension of the concept of Lyapunov exponent, J. Phys. A. Math. Gen., 30, 1-26, 1997.

Aurell E., Boffetta, G., Crisanti, A., Paladin, G., and Vulpinai, A.: Predictability in systems with many degrees of freedom, Phys. Rev. E., 52, 2337, 1996.

Bengtsson, L., Schlese, U., Roeckner, E., Latif, M., Barnett, T. P., and Graham, N.: A two-tiered approach to long-range climate forecasting, Science, 261, 1026-1029, 1993.

Boffetta, G., Crisanti, A., Paparella, F., Provenzale, A., and Vulpiani, A.: Slow and fast dynamics in coupled systems: A time series analysis view, Physica D., 116, 301-312,1998.

Cai, M., Kalnay, E., Toth, Z.: Bred Vectors of the Zebiak-Cane Model and Their Application to ENSO Predictions, J. Climate, 16, 40-56, 2003.

Corazza, M., Kalnay, E., Patil, D. J., Ott, E., Yorke, J., Szunyogh, I., and Cai, M.: Use of the breeding technique in the estimation of the background error covariance matrix for a quasigeostrophic model, in: AMS Symposium on Observations, Data Assimilation and Probabilistic Prediction, Orlando, Florida, 154-157,2002.

Chang, Y., Schubert, S. D., and Suarez, M. J.: Boreal winter predictions with the GEOS-2 GCM: The role of boundary forcing and initial conditions, Quart. J. Roy. Meteor. Soc., 126, 1-29, 2000.

Evans, E., Bhatti, N., Kinney, J., Pann, L., Peña, M., Yang, S.-C., Kalnay, E., Hansen, J.: RISE undergraduates find that regime 
changes in Lorenz's model are predictable, Bull. Amer. Meteor. Soc., 85, 520-524, 2004.

Ji, M., Leetmaa, A., and Kousky, V. E.: Coupled model predictions of ENSO during the 1980s and 1990s at the National Centers for Environmental Prediction, J. Climate, 9, 3105-3120, 1996.

Kalnay, E.: Atmospheric Modelling, Data Assimilation and Predictability, Cambridge University Press, 2003.

Kalnay, E., Corazza, M., and Cai, M.: "Are Bred Vectors the same as Lyapunov Vectors?", extended abstract, http://ams.confex. com/ams/htsearch.cgi, American Meteorological Society meeting, Orlando, Florida 13-17 January 2002, 2002.

Kalnay, E. and Toth, Z.:The breeding method, Proc. of the Seminar on Predictability, ECMWF 4-8 September 1995, ECMWF, Shinfield Park, Reading, Berkshire RG29Ax, e.kooij@ecmwf.int, 69-82, 1996.

Legras, B. and Vautard, R.: A guide to Liapunov vectors, Predictability vol I, edited by Palmer, T., ECMWF Seminar, ECMWF, Reading, UK, 135-146, 1997.

Lorenz, E.: Deterministic non-periodic flow, J. Atmos. Sci., 20, 130-141, 1963.
Lorenz, E.: Predictability - A problem partly solved, Proc. on predictability, ECMWF 4-8 September 1995, ECMWF, Shinfield Park, Reading, Berkshire RG29Ax, e.kooij@ecmwf.int, 1-18, 1996.

Palmer, T., and Anderson, D.: Prospects for seasonal forecasting, Quart. J. Roy. Meteor. Soc., 120, 1026-1029, 1994.

Pegion, P., Shubert, S., and Suarez, M.: Boreal winter predictions with the GEOS-2 GCM: The role of the boundary forcing and initial conditions, Quart. J. Roy. Meteor. Soc., 126, 1-29, 2000.

Timmermann, A.: The predictability of coupled phenomena, Proc. of the seminar on predictability of weather and climate, ECMWF 9-13 September 2002, Reading, England, 2002.

Toth, Z. and Kalnay, E.: Ensemble forecasting at NMC: the generation of perturbations, Bull. Amer. Meteor. Soc., 74, 2317-2330, 1993.

Toth, Z. and Kalnay, E.: Ensemble forecasting at NCEP and the breading method, Mon. Wea. Rev., 125, 3297-3319, 1997.

Yang, S.-C., Kalnay, E., and Cai M.: Bred vectors in the NASA coupled ocean-atmosphere system, Poster presentation, NP81WE2P-1657, EGS Geophys. Res. Abstracts, 5, 12 016, 2003.

Zebiak, S. and Cane, A.: A model for El Niño-Southern Oscillation, Mon. Wea. Rev., 115, 2262-2278, 1987. 